\title{
CONTRIBUTION OF PERVASIVE INTELLIGENCE TO COLLABORATIVE INNOVATION PROCESSES
}

\author{
Veronica Serrano, Thomas Fischer \\ WHU Otto Beisheim School of Management \\ veronica.serrano@whu.edu, fischer@whu.edu
}

Considering the fact that pervasive intelligence will be in foreseeable time a reality, we analyze its influence on collaborative innovation processes and propose a possible innovation system structure. Some ideas about potential ubiquitous systems that could enhance the stages of the innovation process are also presented.

\section{INTRODUCTION}

In a global and technology-oriented world the requirements on products and services increase continuously. In order to face these challenges in $\mathrm{R} \& \mathrm{D}$, different abilities and competencies need to be brought together, e.g., through collaboration with suppliers, customers, external service providers and research institutions, with the objective of achieving high-quality innovations. Innovation processes comprise basically multidisciplinary activities that require disperse teams combining expertise and experience in various fields. Advances in ICT are supporting and making virtual collaboration for innovation feasible. One of the latest paradigms in ICT describes ubiquity of information-processing, communication-technology and computerperformance through embedding sensors, actuators and processors in the environment. It is to expect that this new paradigm will change the organization, the management and even the conception of collaborative innovation processes.

The paper begins describing the new IT paradigm in section 2. Section 3 defines the conceptual structures of pervasive intelligence and the properties of ubiquity that characterize ubiquitous systems. Section 4 presents the possible contributions of pervasive intelligence for enhancing collaborative distributed innovation processes. Finally, section 5 concludes this paper and addresses some potential future trends.

\section{VISIONS OF UBIQUITY}

Three representative conceptions ubiquitous computing, ubiquitous networking and ambient intelligence embody the most important aspects of the vision.

The term ubiquitous computing appeared chronologically at first just before the 
internet boom and the propagation of the computer use (many computers per person) (Weiser, 1993, 1999). Fundamental issues are, e.g. affording ubiquitous operations of everyday artefacts in terms of computation performance and ICT-capabilities. Ubiquitous computing deals with the creation and/or the augmentation of everyday objects to guarantee ubiquitous provision of operation and service for users.

Ubiquitous network refers to the environment for ICT utilization where "a network is connected at any place, at any time and with any object" (Murakami, 2004). Ubiquitous networking (Murakami, Fujinuma, 2000) emphasizes the possibility of building networks of persons and objects for sending and receiving information of all kinds and thus providing the users with services anytime and at any place. This implies to consider: technological aspects, content-based aspects, and organizational aspects by coordinating the formation and development of (often locally dispersed) groups.

Ambient intelligence is the first term that describes the vision as a whole. It concentrates on the arrangement of the architecture, the organization and the coordination of the components (especially ubiquitous, embedded, networked, adaptive applications) of the intelligent environment (ISTAG, 2001). Ambient intelligence aims building structures for an optimal self-organisation of the environment with the objective of enhancing human communication, creating confidence and providing knowledge and skills for a better quality of life (Philips, 2006).

\section{PERVASIVE INTELLIGENCE}

Our conception of pervasive intelligence derives from the integration of ubiquitous computing, ubiquitous networking and ambient intelligence. Etymologically, pervasive intelligence means intelligence that can penetrate everything or is present everywhere. Constitutive characteristics are adaptivity and autonomy. Adaptivity describes the capacity for or tendency toward adaptation, which is the adjustment to environmental conditions or the modification of an organism or its parts that makes it more fit for existence under (externally) emerging conditions. Autonomy represents the quality of self-direction, acting independently of other factors. As constitutive characteristics of pervasive intelligence they describe actions as well as structures.

Adaptivity involves reactive actions to predefined conditions of the environment, to the necessities of other objects as well as to the perceived necessities of specified users in determinate situations Adaptivity also implies changing structures because only they enable the reactivity of the environment as a whole.

Autonomy implicates proactive actions, which take place anticipating future problems, needs of users or possible changes in the environment. Self-configuration and self-organisation are two characteristics of structures to guarantee autonomous behaviour. The configuration, i.e. time, location, activation, varies due to the dynamic incorporation or removal of components or due to changes in the way how persons use or interact with them. Self organising structures are those in which components automatically configure their interaction in a way that is compatible with the remaining interactions in the system (Georgiadis et al., 2002). 
Pervasive intelligence manifests itself in form of ubiquitous systems. Ubiquitous systems are such systems whose components possess properties like contextawareness, embedding and miniaturization as well as availability, reachability and connectivity of sensors, operators and actuators anytime/anywhere and to anything or anybody, invisibility in terms of calm computing, augmented reality and interactivity. A context-aware system is "the system that uses context to provide relevant information and/or services to the user, where relevancy depends on the user's task" (Dey et al., 1999). Miniaturization of sensors, operators and actuators and embedding them into the physical world afford the integration of intelligence (perception, interpretation and reaction-capabilities) into the environments.

One of the greatest challenges of the ubiquitous vision is calm computing that implies supporting human beings in a way that they do not feel disturbed by thousands of different applications and devices that try to guess their needs (Weiser, Brown, 1996). Augmented reality illustrates the integration of the digital and the real world for enhancing the conditions of the environment. Interactivity is another important property of ubiquitous systems. Actions of users and objects actively influence the reactions of the applications and of the environment (two way interaction). This allows the provision of personalized services for the users.

\section{CONTRIBUTION TO COLLABORATIVE INNOVATION}

The potential contribution of pervasive intelligence to collaborative innovation comprises different fields of investigation like the creation of conceptual structures of collaborative innovation processes, the study of already existing and the development of new methodologies as well as the analysis of technological requisites. We concentrate here on the first one: the formulation of innovation system structures considering thereby the information flows of the product lifecycle, the organization and the coordination of innovation processes and the creation and management of knowledge.

\subsection{Innovation System Structure}

Cyclic models represent dynamics and flexibility of innovation processes in a better way than traditional linear ones. Moreover, considering the characteristics of pervasive intelligence, the properties of ubiquitous systems and the interactivity of innovation processes, innovation system structures should be modelled in a cyclical form as outlined in Figure 1.

Three different levels of innovation, i.e. (A) radical, (B) regular or (C) incremental innovations are represented through three cycles.

(A) Radical innovation begins with the stage (1) Identification and Selection, in which the strategic innovation goals are identified and new ideas are screened and generated based on various knowledge resources or basic research. New ideas often result from the iterative collaboration of the design and development team, customers, suppliers and research institutions. Then, the design- and development team structures the ideas and usually supported by customers and suppliers evaluate and select the most feasible, while the useless ones are discarded. Subsequently, the 
selected ideas are passed to the second stage (2) Concept Definition and Project Planning.

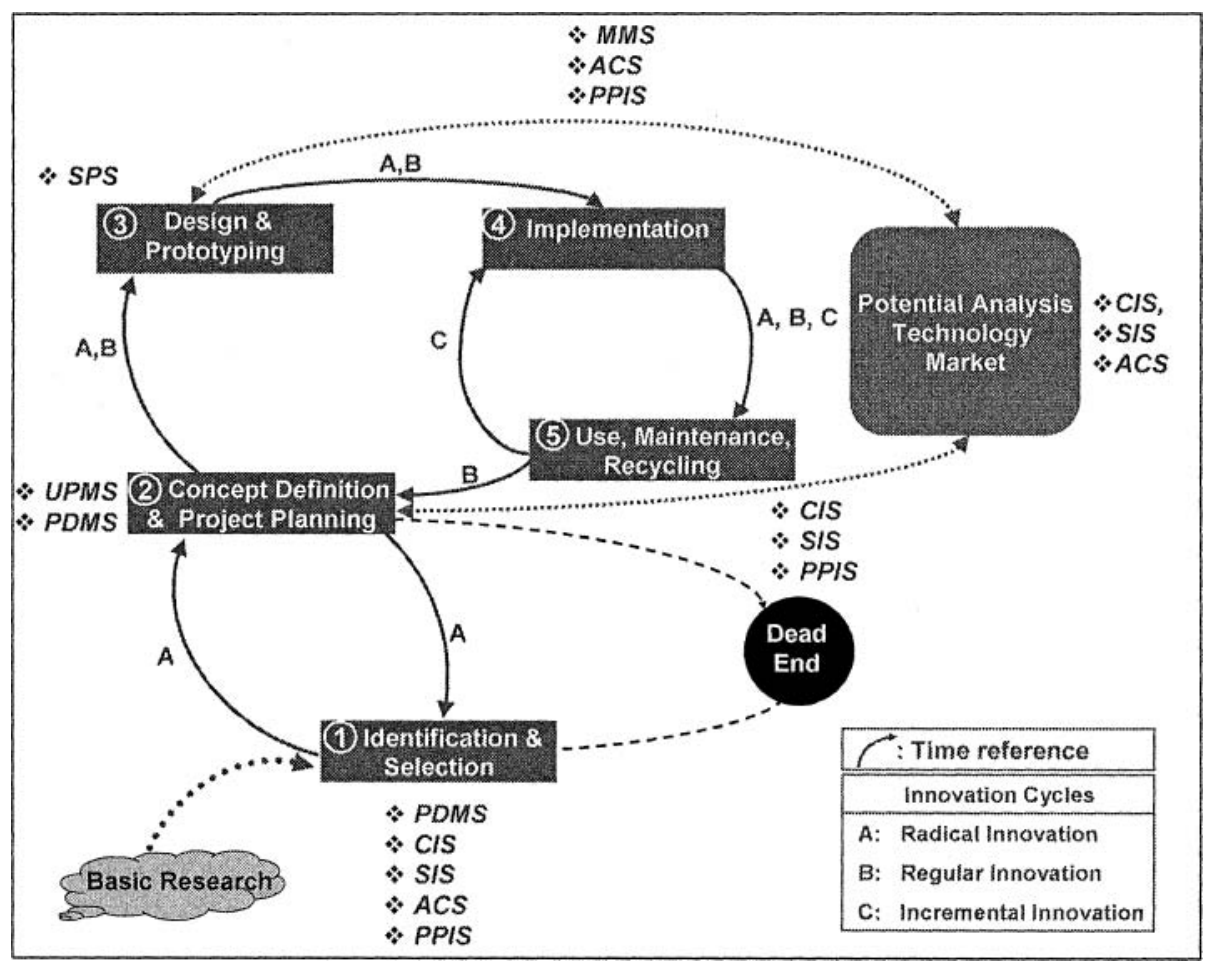

Figure 1 - Innovation System Structure

Combining the selected ideas with the knowledge and the experience of the participants, new concepts are created, e.g., through visualization enabled by augmented reality tools. This also ensures the originality of the transmitted concepts independently of the actual locations of the participants (ubiquity). The validation occurs in form of an analysis of the potential of the new concepts considering their technical feasibility and their market opportunities. The information is between the first and the second stage, rotating from the one to the other until a mature concept is conceived.

Considering the results of the potential analysis, the concept is enhanced and a project plan is set up. Both are sent to the next stage (3) Design and Prototyping where a possible product or process is outlined. This draft serves as basis for the prototype, which is evaluated by customers and technology experts before passing to the next stage. Thus information on possible improvements and especially ideas on new services, which complement the product, are generated

The knowledge and the information generated during the (4) Implementation of the product (service/process) provide a basis for the optimization of the production process. During (5)Use, Maintenance and Recycling the behaviour of the customers is observed applying market studies, surveys or through embedded sensors and (web) interfaces in existing products if they have agreed to send their personal use- 
data. After-market services like maintenance or recycling contribute to continuously discover improvement potential. Information stored in recycled products, brought back to the company, inspires possible changes or new concepts for modification of products, creation of new services or combinations of services and products.

Beyond radical innovation it is possible to differentiate regular and incremental innovation (cycles B and C). Regular innovation (B) takes place when market feedback is remitted to the stage (2) Concept Definition and Project Planning. From there, cycle B starts aiming at the creation of the next generation of products/services passing (3), (4) and (5). Incremental innovation (C), also called continuous improvement, results when the internal and external data are directly transmitted to the stage (4) Implementation in which the noticed recommendations are immediately realized into products or services of the next series.

\subsection{Ubiquitous Innovation Systems}

As indicated in the Figure 1, seven potential ubiquitous systems ${ }^{i}$ can significantly support the stages of the innovation process.

The first stage can be assisted by Pervasive Design Management System (PDMS), Customer Integration System (CIS), Supplier Involvement System (SIS), Ambient Collaboration System (ACS) and Product and Process Information System (PPIS).

PDMS aims at improving efficiency and target orientation avoiding designs that are never used, delivered or completed. The system affords the association of surging ideas with already completed designs in order to prevent possible repetitions of designs. Multimodal interfaces (voice or handwriting recognition) embedded in the physical design environment and interfaces in portable devices (computer, PDA, cell-phone) recognize future possible design purposes during meetings and present similar and useful designs. Thus creativity is stimulated and an efficient selection of ideas is afforded, which contributes to save time and material resources (design rationalisation).

Properties of ubiquity manifested in this system are (in relevance order) contextawareness, embedding and miniaturization, calm computing, interactivity and ubiquitous communication and reachability.

Context-awareness serves for detecting the "design context", i.e. the logical context of the ideas, e.g. using association techniques. Embedded interfaces in the physical environment collect design information on user's demand (calm computing). Interactivity evinces the possibility of communication of the designers with the system. Different features can be selected to conduct various operations. Augmented reality upgrades the quality of information of already designed ideas and projects, e.g., multimedia files explaining the designs or additional information onto the working surface. Ubiquitous communication and reachability play an important role in distributed design meetings, particularly because it is necessary to recognize the context of design in order to localize and contact the necessary expertise or experts. PDMS can be considered an extension of Product Data Management systems that administer all information around products: CAD models, drawings, their associated documents, product visualizations. PDMS includes additionally designs even if they did not result in products and enhances the support to the design team incorporating ubiquitous features. 
CIS intends integrating customers in the innovation process in a more intuitive way. Three different situations of integration are mentioned here: incorporating customers in an artificial environment (living labs), addressing customers directly in the place of consumption or in communal places and forming communities that share similar problems or interests. Real-time communication between the participants of the innovation process facilitates discovering errors and malfunctions as well as solving common problems during operation and application. Recycled products with embedded technology could even gather information about customers' behaviour during the whole period of use.

In CIS the following features are active: context-awareness is necessary for recognizing contexts of use of different customers, embedding and miniaturizing enable the integration of sensing platforms in the environment and in products; calm computing is responsible for not disturbing the customer and not altering the real conditions of use and augmented reality for supporting users by revolving problems during the operation of products. CIS can be understood as specific Customer Relationship Management being a part of a PLM system that integrates various systems along the life cycle (Swink, 2006). Nevertheless, CIS is more specialized because it tries to filter and manages only data that are directly involved in innovation activities.

SIS strives for integrating suppliers early in the innovation process so that real cross-functional team-work is facilitated. Context awareness allows finding adequate suppliers depending on the actual context of the design-task, the designobject, or conditions. Embedded technology and ubiquitous connectivity are responsible for tagging and reaching all the potential (dispersed) suppliers so that even if they are en-route they can be localized or involved through applications in their mobile personal devices. A mixture of context-awareness with ubiquitous connectivity makes possible an intensive but adequate information exchange between suppliers and the rest of participants in the innovation process. The probability of forming open innovation groups as well as unstructured spontaneous meetings could also be increased through context-awareness and ubiquity. All these process should take place in a calm-technology atmosphere so that the suppliers do not feel invaded in their privacy. Augmented reality can enhance the quality of exchanged information. SIS could be part of Supply Chain Management being integrated in a PLM system and concentrating on the collaboration with the supplier in the new product development.

ACS is in charge of the organization and technical realization of successful collaboration in all its forms (co-located and dispersed groups, synchronous or asynchronous, symmetric or asymmetric), which is possible through the coordination of tasks like collecting and exchanging knowledge, distributing experience and skills of multiple team members, etc. Ubiquity permits contacting and localizing the collaborators of the innovation process independent of where they are and respecting what they are doing. Embedded and miniaturized software and hardware augment the capabilities of already used devices and enhance the communication of experts. Context-awareness is responsible for detecting the actual context of the participants so that on the move and without interrupting their main tasks, suppliers, manufacturers, distributors can exchange information at the time that this information surges or when they are available. Ad-hoc collaboration and communication of expertise and experience of extern collaborators (synchronously 
or asynchronously) enrich the work of the innovation team. Augmented reality contributes by exchange of information so that the transmitted ideas or concepts conserve the meaning given by their originators. ACS is a collaborative development system that facilitates the joint work by the development of a product/service representing an enriched version with properties of ubiquity.

PPIS controls the information flows through the product life cycle especially during operations. Sensing and tracking capabilities afford the acquisition of necessary knowledge and information to evaluate production processes. Seamless information flow allows supervising the product life cycle and acquiring ideas of how the manufacturing processes should be improved or innovated. Embedded technology in the machinery, resources and surroundings enables fast diagnostics of errors, which serves as basis for efficient discovery of enhancement potential, and an efficient allocation of resources. Here, the properties embedding and miniaturizing as well as invisibility in terms of calm computing predominate. Through embedded devices the product life cycle can be better monitored but they should remain invisible in order to avoid disturbing the normal operations in manufacturing. The contribution of the system is acquiring knowledge about the product life cycle which is currently very time-consuming and cost-intensive.

The stage (2) Concept Definition \& Project Planning can benefit from the Ubiquitous Project Management System (UPMS) and the PDMS. UPMS like other Project Management Systems is responsible for the organization and management of design projects using information provided by other systems (Material Management Systems (MMS), ACS). Availability of materials can be enquired from any location and an immediate response will be sent to allow dynamic resource allocation and resource mapping (Gajos et al., 2001). Successful project management results from providing easy access to common used services and information. Coordination of appointment calendars that run in different devices and interaction of users for sharing common goods and services represent important goals of this system where ubiquity, interactivity and embedding and miniaturizing predominate. Ubiquity serves to localize the different participants for building distributed virtual organisations in order to provide information about available resources or services. Interactivity is reflected on the constant updating of information about realized tasks, used resources and their interrelations; embedding and miniaturizing of technology enable the collection of lifecycle 's information.

(3)Design and Prototyping can profit from the Smart Prototyping System (SPS), which through embedded sensors, displays or multimodal interfaces in prototypes allows reaching a better quality of the interaction with testers. More transparency, less failure rate and efficiency in the communication among suppliers, marketing staff, manufacturers, partners and in the transmission of opinion, ideas, preferences can be afforded because the prototype transports this information by itself. Experiences with a prototype in specified situations could be recorded and analyzed.

The properties of ubiquitous systems accentuated in SPS are in particular invisibility (through multimodal interfaces) and interactivity to elicit reaction and feedback of the test persons while using prototypes. In addition, context-awareness allows recognizing different behaviours of test-persons according to their actual contexts and embedding and miniaturizing of devices (cameras, sensors) make new forms of obtaining information about the users and their environment more feasible. 
The stage (4) Implementation of a new product or a new product-service combination is influenced by the Material Management System (MMS), the ACS and the PPIS. Supported by, e.g. Auto-ID and RFID technologies physical goods can be localized; information about their state can be provided and understood by machines and this in real time. MMS will enrich and complement Enterprise Resource Planning with properties of ubiquity. Before the new concept is realized as a final product, the feasibility and availability of the crude materials, preliminary products, etc. is controlled for an efficient management of resources.

\section{CONCLUSIONS}

Pervasive intelligence enables a new quality of information sharing, joint planning, joint problem solving, integration of operations, etc. These factors will positively influence collaborative innovation processes. Ubiquitous innovation systems will increase the performance of the whole innovation cycle.

In a near future it is even conceivably that autonomy and adaptivity develop in such a way that a system could even open up unexpected knowledge-sources independently in order to satisfy the needs of actors in the innovation environment. This will be the next step and is what we call "Emergent Intelligence".

\section{REFERENCES ${ }^{\text {ii }}$}

1. Dey A, Salber D, Abowd G. "The Conference Assistant Combining Context-awareness with Wearable Computing". GCU Center, Georgia Institute of Technology, Atlanta, 1999.

2. Gajos K, Weisman L, Shrobe H. "Design principles for Resource Management Systems for Intelligent Spaces". Draft for the Workshop on Self-Adaptive Software, Budapest, Hungary, 2001.

3. Georgiadis I, Magee J, Kramer J. "Self-Organising Software Architectures for Distributed Systems". WOSS 02 in ACM, 2002.

4. ISTAG, "Scenarios for Ambient Intelligence in 2010". http://www.cordis.lu/ist/istag.htm, 2001.

5. Murakami T, Fujinuma A. "Ubiquitous Networking: Towards a New Paradigm". Nomura Research Institute Papers No. 2, 2000.

6. Murakami, Teruyasu. "Ubiquitous Networking: Business Opportunities and Strategic Issues". Nomura Research Institute Papers No. 79, 2004.

7. Philips Research Webpage stand: January 2006 http:/www.research.philips.com/technologies/syst_softw/ami/vision.html .

8. Swink, Morgan. "Building collaborative innovation capability". In Research-Technology Management, Volume 49, Number 2, March-April 2006, pp. 37-47(11).

9. Weiser, Mark "Some Computer Science Issues in Ubiquitous Computing". In Communications of ACM, Vol. 36, No. 7, 1993.

10. Weiser M, Brown J. "The Coming Age of Calm Technology". Xerox PARC, 1996.

11. Weiser, Mark. "The computer of the $21^{\text {st }}$ century". In ACM SIGMOBILE, Vol. 3, Issue 3, 1999.

\footnotetext{
${ }^{\mathrm{i}}$ In the following the features of ubiquity and in particular pervasive intelligence are addressed in a very compressed form due to the restricted space of the paper. These systems are under current research of the authors.

ii The presented ideas are based on a number of papers that cannot be referred in detail due to the restricted space.
} 